\title{
Avaliação pós-ocupação de unidades de emergência hospitalares de Florianópolis: problemas recorrentes e possíveis soluções
}

\author{
Post-occupancy evaluation of emergency units in \\ Florianópolis hospitals: recurring problems and possible \\ solutions
}

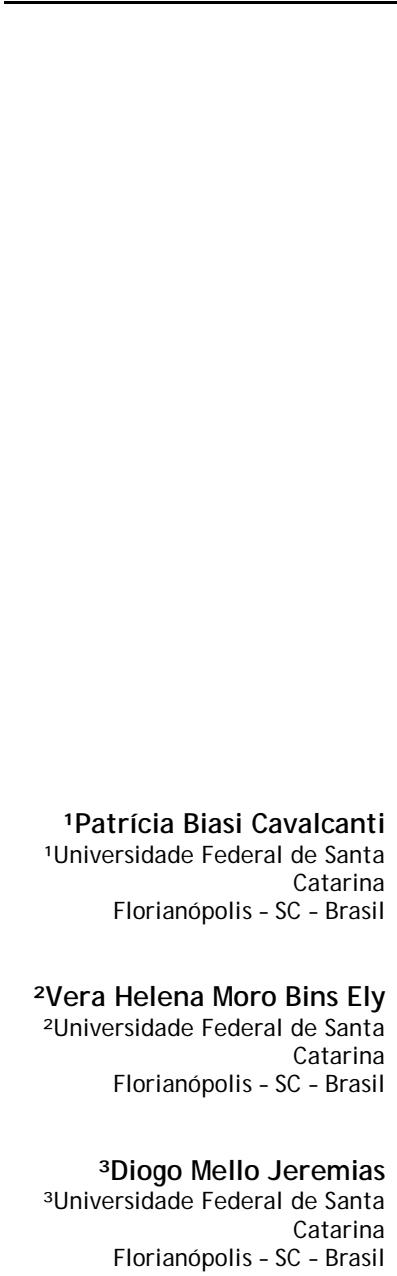

\author{
Patrícia Biasi Cavalcanti \\ Vera Helena Moro Bins Ely \\ Diogo Mello J eremias \\ J oão Pedro Schneider \\ Marcus Vinícius da Silva \\ Thaize Vanessa Costa Bortoluzzi
}

\section{Resumo}

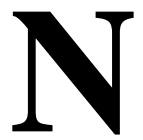

este trabalho buscou-se refletir sobre a configuração espacial de unidades de urgência e emergência por meio da avaliação de seis destas unidades situadas em hospitais públicos da Grande

Florianópolis, SC. A metodologia incluiu: revisão de literatura, visitas exploratórias e seis estudos de caso. Nos estudos de caso, foi aplicado o método Walkthrough por meio de dois amplos checklists, sendo o primeiro referente a aspectos mínimos necessários ao funcionamento da unidade e dos ambientes, e o segundo relativo à sua humanização. Os checklists totalizavam aproximadamente 500 itens avaliados, abrangendo a relação entre os usuários e o ambiente hospitalar, sua percepção e comportamento ambiental. Dentre os resultados constatados destacaram-se: subdimensionamento de ambientes, sobreposição de usos, inadequação do leiaute às atividades realizadas, problemas de acessibilidade, ausência de alguns ambientes necessários ao local e problemas de manutenção. $\mathrm{O}$ artigo reflete ainda sobre algumas possíveis soluções para esses problemas, especialmente aquelas referentes à atuação dos arquitetos e ao processo de revisão da Resolução RDC 50/2002.

Palavras-chave: Arquitetura hospitalar. Unidades de urgência e emergência. Avaliação pós-ocupação. Percepção ambiental.

\section{Abstract}

The aim of this paper is to reflect on the spatial configuration of urgency and emergency units through the evaluation of six of them situated in public hospitals in the metropolitan area of Florianópolis, SC, Brazil. The methodology includes:

${ }^{4}$ oão Pedro Schneider ${ }^{4}$ Universidade Federal de Santa Catarina Florianópolis - SC - Brasil

${ }^{5}$ Marcus Vinícius da Silva 5Universidade Federal de Santa Catarina Florianópolis - SC - Brasil

${ }^{6}$ Thaize Vanessa Costa Bortoluzzi ${ }^{6}$ Universidade Federal de Santa Catarina Florianópolis - SC - Brasil

Recebido em 22/05/18 Aceito em 06/08/18 literature review, exploratory visits and six case studies. In the case studies, the Walkthrough Analysis was applied via two comprehensive checklists, the first one concerning the minimum aspects necessary to the functioning of the unit and its environment, and the second one concerning its environmental human scale. The two checklists totalled nearly 500 evaluated items, covering the relationship between users and the hospital setting, their environmental perception and behaviour. The results highlighted: undersized environments, overlapping of uses, inadequacy of the layout to the activities carried out, accessibility problems, lack of certain necessary environments and maintenance problems. The article also discusses possible solutions to these problems, especially those related to the architect's performance and to RDC 50/2002 reviewing process.

Keywords: Healthcare architecture. Urgency and emergency units. Post occupancy evaluation. Environmental perception.

CAVALCANTI, P. B.; BINS ELY, V. H. M.; J EREMIAS, D. M.; SCHNEIDER, J. P.; SILVA, M. V. da; BORTOLUZZI, T. V. C. Avaliação pós-ocupação de unidades de emergência hospitalares de Florianópolis: problemas recorrentes e possíveis soluções. Ambiente Construído, Porto Alegre, v. 19, n. 2, p. 171-186, abr./ jun. 2019.

ISSN 1678-8621 Associação Nacional de Tecnologia do Ambiente Construído. http:// dx. doi.org/ 10.1590/ s1678-86212019000200315 


\section{Introdução}

As unidades de urgência e emergência de hospitais públicos apresentam-se como um dos principais meios de acesso da população ao atendimento no Sistema Único de Saúde (SUS) no intuito de dispor de assistência médica imediata que dispense o agendamento prévio. Assim, com frequência, as unidades de urgência ou emergência são procuradas mesmo quando os pacientes não constituem casos dessa natureza, o que tende a gerar quadros de superlotação. Como consequência, podem ser comuns os conflitos entre pacientes, acompanhantes e profissionais de saúde, quando não há a compreensão pelo público de que os casos menos graves tendem a esperar durante horas por atendimento, enquanto os casos mais graves recebem atendimento prioritário.

A Portaria n. 2.048 (BRASIL, 2002) reafirma a importância das unidades de urgência e emergência como componente da assistência à saúde e atribui a crescente demanda de pacientes ao aumento no número de acidentes e da violência urbana. Por sua complexidade e importância para o funcionamento do sistema de saúde, as unidades de urgência e emergência do país têm sido tema constante de discussão e reflexão no âmbito dos órgãos e gestores públicos. Embora haja legislação regulamentando o funcionamento e o planejamento das unidades de urgência e emergência no país, esta nem sempre é plenamente cumprida.

Nesse contexto de frequente superlotação das unidades de urgência e emergência tem-se um impacto negativo em sua percepção pela população, o que a torna tema recorrente na mídia. As soluções para o problema permeiam ações e políticas públicas, mas também aspectos relativos à sua configuração ambiental e ao âmbito da arquitetura.

O presente trabalho busca contribuir para a discussão do tema, por meio da avaliação exploratória e sistemática de seis unidades de urgência e emergência de hospitais públicos da Grande Florianópolis, Santa Catarina. Buscou-se identificar problemas recorrentes na configuração espacial dessas unidades e refletir sobre possíveis soluções. A metodologia incluiu: revisão de literatura, visitas exploratórias a duas unidades de urgência e emergência e a realização de seis estudos de caso. Os estudos de caso centram-se em uma aprofundada análise Walkthrough, com a aplicação de dois checklists que totalizam aproximadamente 500 itens de avaliação, respondidos pelos pesquisadores e pelos profissionais de saúde que atuam no local.

\section{Fundamentação teórica}

No Brasil, a organização dos serviços de saúde se consolidou de forma heterogênea, variando segundo fatores como: modelo tecnológico, tipo de sistema - privado, filantrópico, previdenciário ou estatal - e ainda de acordo com os níveis assistenciais - atenção básica, secundária ou terciária (POLL; LUNARDI; LUNARDI FILHO, 2008). O Ministério da Saúde propõe que haja uma hierarquia no atendimento aos cidadãos de acordo com os níveis de complexidade (BRASIL, 2006). A rede de atenção básica, que inclui, por exemplo, os postos de saúde, deveria receber a maior parcela da população que procura atendimento, uma vez que nela se praticam ações de educação, de prevenção e de promoção da saúde. Esta seria uma prática coerente com a compreensão atual do que é saúde, entendida não apenas como tratamento de doenças, mas como a preservação do bem-estar físico, mental e social do indivíduo (ORGANIZAÇÃO..., 1978). Nessa perspectiva, apenas os casos de segundo e terceiro nível de complexidade seriam encaminhados para a rede hospitalar.

As unidades de urgência e emergência, por sua vez, proporcionariam atendimento imediato a: pacientes com risco de vida, casos de emergência, sendo esse serviço considerado de alta complexidade; e pacientes sem risco de vida, casos de urgência, sendo esse serviço classificado em baixa e média complexidade ou em alta complexidade (AGÊNCIA..., 2002). As unidades de urgência podem direcionar-se ao atendimento de baixa e média complexidade, como é o caso das Unidades de Pronto Atendimento (UPAs), que constituem serviços extra-hospitalares. As unidades de urgência e emergência direcionam-se ao atendimento de alta complexidade quando, por exemplo, enquadram-se no nível terciário de atenção, em geral correspondendo àquelas que se situam em hospitais (GÓES, 2006).

$\mathrm{O}$ acesso do paciente ao atendimento público de saúde no país se dá principalmente por unidades de atenção básica e por serviços de urgência e emergência (ALMEIDA et al., 2000). No entanto, grande parte das pessoas que procuram unidades de urgência e emergência constitui-se em casos que poderiam receber atendimento no nível de atenção básica. A preferência por essas unidades deve-se à possibilidade de dispor de um diagnóstico e tratamento mais rápidos, tendo em vista alguns problemas de acesso e resolutividade da rede de atenção básica (AZEVEDO et al., 2010). Além disso, a preferência pelas unidades de urgência e emergência também ocorre por influência de fatores sociais, epidemiológicos e

172 Cavalcanti, P. B.; Bins Ely, V. H. M.; J eremias, D. M.; Schneider, J. P.; Silva, M. V. da; Bortoluzzi, T. V. C. 
macroeconômicos (ACOSTA; LIMA, 2015; OLIVEIRA; PINTO, 2015).

Assim, a dificuldade da rede pública de saúde brasileira em acolher e atender a população em contínua expansão (POLL; LUNARDI; LUNARDI FILHO, 2008) contribui para o aumento da demanda de atendimento nas unidades de urgência e emergência, ocasionando problemas de superlotação (FATOVICH, 2002). Esse é um problema internacional que se agrava, e que em alguns países é o principal fator que afeta a qualidade dos serviços prestados por essa unidade (FATOVICH, 2002; DERLET; RICHARDS, 2000).

Nas causas da superlotação dessas unidades relativas à estruturação da rede de saúde incluemse: anseio por melhores recursos tecnológicos, facilidade de acesso a medicamentos (PUCCINI; CORNETTA, 2008), funcionamento 24 horas por dia, desconhecimento dos níveis de complexidade de atendimento por grande parte da população, e, ainda, indefinição do diagnóstico (GIACOMO, 2011). Também se destaca a dificuldade de encaminhar pacientes já atendidos por unidades de urgência e emergência para outros setores ou serviços de saúde do próprio hospital ou de outros estabelecimentos para efetuar: exames adicionais, avaliação de peritos ou internação (DESLANDES et al., 2006).

Nas causas da superlotação das unidades de urgência e emergência relativas ao perfil epidemiológico e demográfico estão: a demanda ambulatorial oriunda de cidades vizinhas menos equipadas (DESLANDES et al., 2006), a localização da unidade próxima a local de moradia ou trabalho (GIACOMO, 2011), e os elevados índices do país de causa externa de morte - não natural - como violência urbana (GAWRYSZEWSKI; KOIZUMI; MELLOJORGE, 2004). Além disso, a elevada prevalência de idosos e de pessoas portadoras de doenças crônicas nessas unidades pode indicar a vulnerabilidade desses indivíduos na demanda da assistência à saúde (ACOSTA; LIMA, 2015) ou ainda ser decorrente do acentuado envelhecimento populacional ocorrido nas últimas décadas (BRASIL, 2013).

Como consequência da superlotação tem-se: a queda na qualidade do serviço prestado; a insuficiência de recursos financeiros, tecnológicos e humanos frente à demanda; e o não atendimento às expectativas do público (TOLEDO; FERRER, 2004). Paul, Reddy e Deflitch (2010) apontam ainda implicações como: problemas de comunicação entre os usuários, longo tempo de espera, pacientes sem atendimento ou sendo atendidos nos corredores, elevados níveis de estresse, diminuição da capacidade da unidade em responder a acidentes em massa, diminuição da motivação e produtividade dos funcionários, e aumento da probabilidade de erros médicos. Poll, Lunardi e Lunardi Filho (2008) apontam ainda que as elevadas taxas de ocupação dos leitos podem levar à falta de privacidade pela redução de espaço entre eles, decorrente da limitação de área disponível.

Sendo assim, arquitetos se deparam com uma problemática desafiadora ao planejarem unidades de urgência e emergência. Zilm (2007) descreve que essa é uma das áreas hospitalares mais complexas, que cresce mais rapidamente e que exige dos provedores que considerem: o gerenciamento de pacientes de baixa gravidade que procuram o local, a capacidade para enfrentar surtos epidêmicos e a necessidade de integrar cuidados centrados nos pacientes. Para o seu planejamento e dimensionamento, Pascale et al. (2014) destacam que a maioria dos guias internacionais baseia-se em médias de atendimentos anuais. No entanto, segundo os autores, costuma haver uma alta variabilidade da demanda, com grande flutuação do volume de pacientes em diferentes épocas do ano e circunstâncias, como a maior incidência de doenças respiratórias no período de inverno. Segundo os autores, essa flutuação da demanda deveria ser considerada nos projetos, não apenas os valores médios anuais.

Zilm (2007) aponta ainda diversas estratégias projetuais que deveriam ser adotadas no planejamento dessas unidades para que se possa atender adequadamente a população em situações extremas e inesperadas, como em caso de desastres ou epidemias. Entretanto, possivelmente devido a restrições econômicas, a maioria dos hospitais atualmente não atende a todas as estratégias projetuais sugeridas, tornando necessário otimizar recursos existentes (PAUL; REDDY; DEFLITCH, 2010). Em algumas cidades dos Estados Unidos, hospitais independentes, distantes geograficamente de hospitais de referência, estão sendo reformados com o intuito de descentralizar cada vez mais o atendimento, aliviando a demanda dos grandes complexos hospitalares. Também nos Estados Unidos têm sido criadas clínicas médicas para atendimento de urgência e têm-se buscado modos de racionalizar a triagem dos pacientes, com avaliações mais rápidas nos horários de pico (ZILM, 2007).

A compreensão desse contexto mostra-se relevante para o planejamento de unidades de urgência e emergência mais adequadas às necessidades de seus usuários. Embora a maioria dos problemas 
descritos não seja do âmbito arquitetônico, eles refletirão diretamente no planejamento e na vivência dos ambientes. Além disso, inúmeros estudos confirmaram o importante papel desempenhado pelo ambiente hospitalar no que se refere à qualidade do atendimento (RISMANCHIAN; HOON LEE, 2017). Assim, acredita-se que a compreensão dos problemas frequentes nessas unidades pode dar suporte à busca por novas soluções e estratégias arquitetônicas.

O objetivo principal deste trabalho consiste em identificar problemas recorrentes relativos à configuração espacial de unidades de urgência e emergência hospitalares e refletir sobre possíveis soluções, sejam elas do âmbito da arquitetura, da gestão pública ou da revisão da resolução RDC 50/2002 (AGÊNCIA..., 2002). São objetivos secundários da pesquisa: compreender o contexto de funcionamento e a problemática relativa à configuração espacial de unidades de urgência e emergência hospitalares; elaborar um instrumento (checklist) de fácil utilização e que permita avaliar unidades existentes, por profissionais de arquitetura, engenharia ou gestores, facilitando a identificação de quais delas demandam ações de manutenção, reforma ou ampliação com maior urgência; contribuir para o planejamento de futuras unidades, por meio desse mesmo instrumento (checklist), uma vez que ele também pode ser usado para conferir se os critérios mínimos desejáveis ao local estão sendo contemplados pelo projeto.

\section{Metodologia}

A pesquisa tem abordagem qualitativa, exploratória e multimétodos, e se enquadra na linha de avaliação pós-ocupação (APO). Centra-se na compreensão da relação entre os usuários e o ambiente hospitalar, e em especial em sua percepção ambiental.

$\mathrm{O}$ estudo foi estruturado em quatro etapas. $\mathrm{Na}$ primeira etapa foi realizada a revisão de literatura, e nela buscou-se compreender: o funcionamento das unidades de urgência e emergência, as atividades nela realizadas, o perfil dos seus usuários, sua configuração ambiental, e a temática de humanização.

$\mathrm{Na}$ segunda etapa foram realizadas visitas exploratórias em duas unidades de urgência e emergência de Florianópolis: uma situada em um grande hospital público federal e a outra em uma unidade privada de atendimento recentemente reformada. Com as visitas exploratórias, buscou-se aproximar os pesquisadores em relação ao tema de estudo, e ainda conhecer diferentes possibilidades de configuração ambiental da unidade. Nessa etapa foi possível: percorrer os ambientes juntamente com o responsável por cada unidade, entrevistá-lo, fazer observações diretas e sistemáticas e realizar registros fotográficos.

A terceira etapa correspondeu aos estudos de caso realizados em seis unidades de urgência e emergência públicas da Grande Florianópolis, cinco delas geridas pela Secretaria Estadual da Saúde. Tendo em vista o número de estudos de caso que se pretendia realizar, a baixa disponibilidade de tempo da equipe de saúde para participar da pesquisa e a intenção de poder comparar os resultados obtidos nas diferentes unidades, optou-se por utilizar um único método a análise Walkthrough. Com o método, buscou-se realizar uma aplicação relativamente rápida, por meio de um roteiro abrangente, e que refletisse tanto o ponto de vista dos pesquisadores quanto da equipe de saúde, uma vez que os usuários eram entrevistados à medida que se percorriam todos os ambientes.

Assim, para fins deste trabalho, e visando também sua aplicação em avaliações futuras, foram desenvolvidos dois checklists pela equipe de pesquisa. O formato adotado nos checklists se baseia nos instrumentos do National Health Service (NATIONAL..., 2005a, 2005b): ASPECT (A Staff and Patient Environment Calibration Toolkit) e AEDET Evolution (Achieving Excellence Design Evaluation Toolkit). Esses dois instrumentos foram criados na Inglaterra com o propósito de avaliar a qualidade da arquitetura hospitalar no que se refere a aspectos gerais e de humanização e não se restringem a unidades de urgência e emergência. A adoção desses instrumentos internacionais como referencial para os checklists desenvolvidos deve-se à sua facilidade de compreensão, pois se estruturam em perguntas claras e objetivas, cujas respostas geram uma pontuação que facilita a comparação entre diferentes unidades avaliadas. Assim, desses instrumentos ingleses adotou-se, sobretudo, o modo simples de apresentação dos itens a serem avaliados e a possibilidade de atribuir pesos e notas. Porém, no que se refere ao conteúdo, o primeiro checklist criado para esta pesquisa, e também o mais extenso, diferencia-se dos formulários ingleses, pois não se centra em avaliar a excelência da arquitetura hospitalar, mas sim os aspectos mínimos a serem contemplados pelos ambientes de unidades de urgência e emergência, incluindo as questões técnicas previstas na Resolução RDC 50/2002 (AGÊNCIA..., 2002) e na Norma NBR 9050 (ABNT, 2004, 2015), além de outros aspectos considerados indispensáveis à realização das atividades dos usuários (Figura 1).

174 Cavalcanti, P. B.; Bins Ely, V. H. M.; J eremias, D. M.; Schneider, J. P.; Silva, M. V. da; Bortoluzzi, T. V. C. 
Já o segundo checklist, menos extenso e avaliado separadamente do anterior, é relativo a aspectos desejáveis ao local como: acessos, comunicação da informação arquitetônica (legibilidade, orientabilidade e sinalização), humanização e conforto ambiental (Figura 2). Esse segundo checklist apresenta um perfil de perguntas e de assuntos próximo aos conteúdos do ASPECT e AEDET, porém abarcando apenas questões de interesse de unidades de urgência e emergência, e com uma menor quantidade de itens. Juntos, os dois checklists totalizam aproximadamente 500 perguntas, sendo a maioria delas organizadas por ambientes avaliados. Assim, embora se tenha optado por um instrumento único de avaliação, buscou-se assegurar uma avaliação abrangente e completa das unidades e de cada um dos seus ambientes, ainda que em caráter exploratório, e que evidenciasse tanto a percepção da equipe de pesquisadores quanto dos usuários do local (JEREMIAS et al., 2017).

Por meio da pontuação final, os checklists possibilitam tanto identificar ambientes mais problemáticos de cada unidade, como comparar diferentes unidades entre si, dando suporte a gestores públicos na identificação de quais delas demandariam obras de reforma com maior urgência, por exemplo.

\section{Figura 1 - Parte do checklist 1, referente aos aspectos mínimos necessários aos ambientes que compõem a unidade}

\begin{tabular}{|c|c|c|c|c|c|}
\hline Área de Desembarque de Ambulância (não-crítico) & Lei & Peso & Nota & Nota final & Tipo de resposta \\
\hline Possui a área mínima de acordo com a RDC $50\left(21 \mathrm{~m}^{2}\right.$ de área coberta)? & RDC 50 & 2 & 1 & 2 & $s / n$ \\
\hline O ปão da porta assegura acesso f́cil ao paciente $(1,10 \times 2,10 \mathrm{~m})$ ? & RDC 50 & 1 & 1 & 1 & $s / n$ \\
\hline A porta possui maçaneta do tipo alavanca? & NBR 9050 & 1 & 1 & 1 & $s / n$ \\
\hline $\begin{array}{l}\text { As dimensões do ambiente e as circulações estão adequadas para as atividades } \\
\text { (desembarque da ambulância e acesso à edificaçâo)? }\end{array}$ & & 2 & 1 & 2 & $s / n /$ parcial \\
\hline $\begin{array}{l}\text { Existe uma cobertura adequada que proteja das intempéries o desem barque da } \\
\text { ambulância? }\end{array}$ & $\mathrm{RDC} 50$ & 2 & 1 & 2 & $s / n$ \\
\hline $\begin{array}{l}\text { Possui revestimento de piso adequado (nivelado, antiderrapante, uniforme e sem } \\
\text { obstáculos)? }\end{array}$ & $\mathrm{RDC} 50$ & 1 & 1 & 1 & $s / n$ \\
\hline $\begin{array}{l}\text { O ambiente está próximo de áreas com atividades afins (Sala de Emergência, Sala } \\
\text { de Procedimentos Especiais, Sala de Higienização)? }\end{array}$ & & 1 & 1 & 1 & $s / n /$ parcial \\
\hline \multirow[t]{2}{*}{ O ambiente encontra-se em bom estado de manutenção? } & & 1 & 1 & 1 & $s / n /$ parcial \\
\hline & & 11 & & 11 & \\
\hline
\end{tabular}

Figura 2 - Parte do checklist 2, referente a um dos aspectos desejáveis para assegurar a qualidade da arquitetura

\begin{tabular}{|c|c|c|c|c|c|}
\hline Comunicação visual & Lei & Peso & Nota & Nota final & $\begin{array}{l}\text { Tipo de } \\
\text { resposta }\end{array}$ \\
\hline A entrada e a saida da U nidade são facilmente identifcadas? & & 2 & 1 & 2 & \\
\hline Existe a informação sobre 0 atendimento por niveis de gravidade? & & 2 & 1 & 2 & $s / n$ \\
\hline $\begin{array}{l}\text { Existem placas com letras grandes e de cor contrastante que indiquem o caminho } \\
\text { a seguir para chegar aos demais ambientes do edifício, como banheiros, recepção, } \\
\text { consultórios, etc.? }\end{array}$ & & 2 & 1 & 2 & $s / n$ \\
\hline $\begin{array}{l}\text { Junto às portas de cada ambiente, existem placas com letras em relevo ou em } \\
\text { Braille na altura entre } 90 \text { e } 110 \text { centimetros que identifiquem seu uso para pessoas } \\
\text { com deficiência visual? }\end{array}$ & & 1 & 1 & 1 & $s / n$ \\
\hline $\begin{array}{l}\text { Na recepção, existe Mapa Tátil que possibilite ao usuário com deíciência visual } \\
\text { localizar-se, identificando o local das diferentes atividades e definindo os caminhos } \\
\text { a seguir? }\end{array}$ & & 1 & 0 & 0 & $s / n$ \\
\hline $\begin{array}{l}\text { Na existência de Mapa Tátil, há piso tátil direcional que guie os defíentes visuais } \\
\text { até a seu destino? }\end{array}$ & & 1 & 0 & 0 & $s / n$ \\
\hline $\begin{array}{l}\text { Existe um seriço de atendimento para pessoas com deficiência auditiva, ou } \\
\text { surdo/cegas, prestado por pessoas capacitadas - intérpretes de Libras - ou por } \\
\text { um equipamento de tecnologia assistiva, como um computador? }\end{array}$ & & 2 & 1 & 2 & \\
\hline $\begin{array}{l}\text { Como você avaliaria a questão dos fuxos intemos entre os ambientes da unidade } \\
\text { de Emergência? }\end{array}$ & & 2 & 1 & 2 & opinião \\
\hline Como você avaliaria a totalidade dos ambientes da Unidade de Emergência & & 2 & 1 & 2 & opinião \\
\hline $\begin{array}{l}\text { Como você avaliaria a localização da Unidade de E mergência dentro do hospital } \\
\text { (facilidade de acesso a pacientes de ambientes extemos e setores afim) }\end{array}$ & & 2 & 1 & 2 & opinião \\
\hline \multicolumn{3}{|c|}{ Os usuários conseguem se orientar dentro da Unidade sem auxílio dos funcionários do Hospital? } & 1 & 0 & opinião \\
\hline $\begin{array}{l}\text { Como você avaliaria a legibilidade dos am bientes (facilidade de reconhecer a que } \\
\text { se destina aos ambientes; portas de entrada, contraste piso/parede, contraste de } \\
\text { acabamentos, entre ambientes) }\end{array}$ & & 2 & 1 & 2 & opinião \\
\hline & & 19 & & 17 & \\
\hline
\end{tabular}


Tendo em vista a extensão dos checklists e a pouca disponibilidade de tempo da equipe de saúde, todos os itens que eram diretamente observáveis ou diziam respeito às resoluções e normas foram respondidos pelos pesquisadores. Por exemplo, os próprios pesquisadores checavam e respondiam se a área do ambiente atendia ao mínimo previsto na Resolução RDC 50/2002 (AGÊNCIA..., 2002), conferindo na planta baixa e in loco. Já as questões que demandavam uma compreensão aprofundada da vivência do local foram respondidas pelos profissionais de saúde, e eram diferenciadas por outra cor de fundo na planilha. Como exemplos incluem-se as perguntas relativas à adequação do leiaute e área de circulação às atividades que ocorriam em cada ambiente. As perguntas respondidas pela equipe de saúde em geral receberam um peso maior do que a maioria das perguntas referentes aos critérios técnicos, tendo em vista sua relevância, bem como o intuito de que a avaliação fosse centrada no usuário. Em todas as unidades ao menos um profissional da chefia médica ou de enfermagem acompanhava toda a avaliação, respondendo ao checklist enquanto se percorria o local. Após o término da aplicação do checklist com o profissional da chefia era solicitada a permanência no local para investigar a percepção ambiental dos demais profissionais de cada ambiente específico, por meio de uma segunda aplicação das perguntas do checklist. Por exemplo, perguntava-se à farmacêutica sobre sua percepção ambiental em relação à farmácia satélite, quando existente, complementando a resposta já fornecida pelo profissional da chefia da unidade. Assim, em cada uma das unidades avaliadas participou um número variado de profissionais de saúde, incluindo todos aqueles que tivessem disponibilidade de tempo para contribuir para a avaliação. Dessa forma, os dois checklists permitiram registrar de forma sistemática observações diretas de aspectos técnicos e objetivos do ambiente, e informações verbais relativas à percepção dos usuários e seu nível de satisfação em relação ao local. Juntamente com a aplicação dos checklists era realizado o registro fotográfico de todos os ambientes da unidade. No trabalho de campo, buscou-se ainda sempre dispor da participação de ao menos quatro dos integrantes da equipe de pesquisa, ficando um responsável por fazer as perguntas, um pelas fotografias, e os demais por anotar as respostas e os dados levantados.
Anteriormente à coleta dos dados foi realizado um estudo-piloto em uma unidade de urgência e emergência de um hospital público de Florianópolis, com o intuito de testar e aprimorar os checklists criados. Em seguida, já havendo readequado os checklists, os pesquisadores realizaram as avaliações em outras cinco unidades de urgência e emergência públicas situadas na Grande Florianópolis, de média a alta complexidade. Apenas uma das unidades analisadas atende exclusivamente crianças, as demais atendem todas as faixas etárias. Como houve pouquíssimas alterações do checklist posteriormente à aplicação do estudo-piloto, e em geral essas alterações foram relativas à forma e não ao conteúdo das questões, os resultados obtidos na primeira aplicação foram considerados nos resultados finais deste trabalho, totalizando as seis unidades avaliadas.

Finalmente, na quarta etapa foi realizada a análise e o tratamento dos dados a partir de uma matriz de descobertas (RHEINGANTZ et al., 2009) para cada uma das unidades analisadas, permitindo sintetizar e representar espacialmente os principais resultados, de forma a facilitar sua compreensão. Assim, os dados de cada ambiente foram organizados por meio de textos sintéticos, associados a imagens e à planta baixa da unidade (Figura 3).

Por fim, foram ainda elaboradas duas tabelas síntese referentes aos resultados dos dois checklists aplicados nas seis unidades. Na primeira tabela, referente ao checklist 1 , as colunas numeram as unidades hospitalares analisadas, e as linhas organizam os resultados por ambientes (Figura 3). Na segunda tabela, referente ao checklist 2, as colunas também numeram as unidades hospitalares, e as linhas correspondem aos quatro itens analisados - acessos, comunicação visual, conforto ambiental e humanização. Os resultados dispostos nas tabelas foram organizados por meio de categorias elaboradas com análise de conteúdo e dizem respeito a: subdimensionamento; leiaute; sobreposição de usos; programa de necessidades; acessibilidade; e estado de conservação ou manutenção. Assim, com o auxílio da tabela foi possível identificar os problemas mais recorrentes nas unidades de urgência e emergência em geral e por ambientes específicos. 
Figura 3 - Imagem da matriz de descobertas de uma das unidades avaliadas

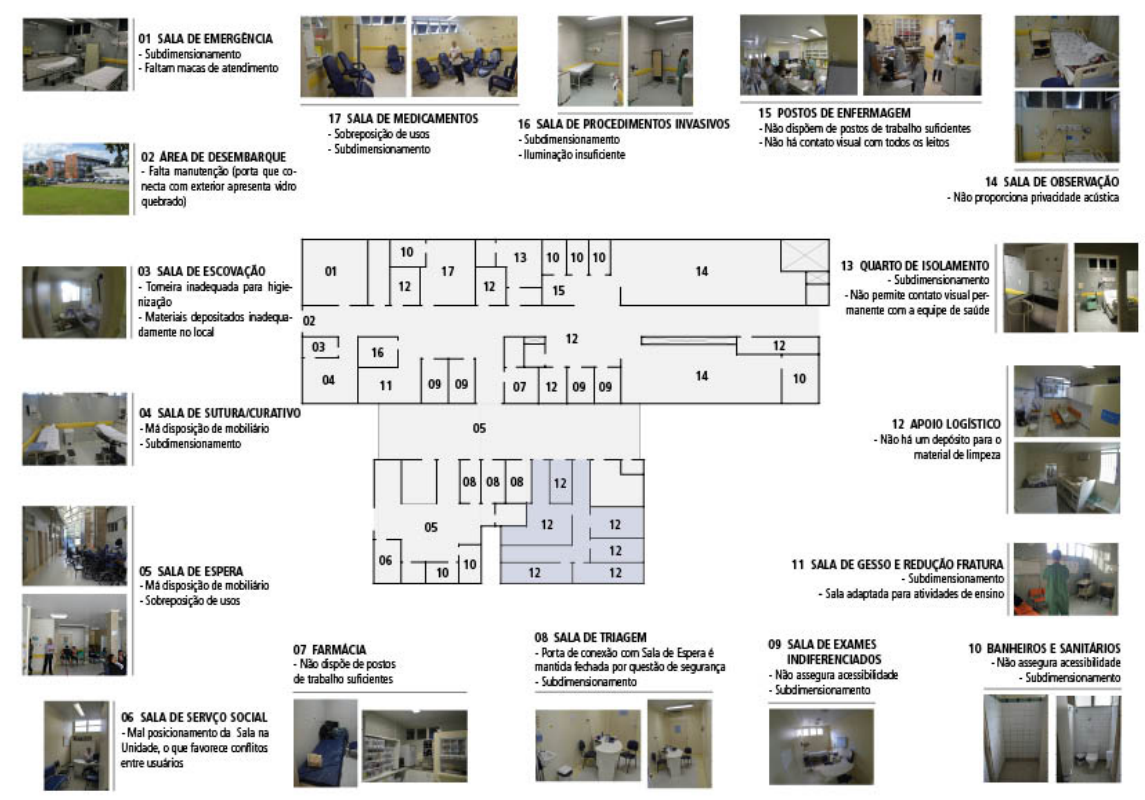

\section{Análise e discussão dos resultados}

Encontra-se a seguir parte da tabela com a síntese dos resultados obtidos nas seis unidades de urgência e emergência avaliadas (Figura 4), seguida da análise e discussão. As instituições de saúde não são identificadas, tendo em vista o compromisso assumido de preservar seu anonimato.

A partir das tabelas síntese dos resultados pode-se observar que os problemas mais recorrentes nas unidades avaliadas referem-se a: subdimensionamento de ambientes frente à área mínima prevista na Resolução RDC 50/2002 (AGÊNCIA..., 2002) ou frente à atual demanda de atendimento; problemas relativos ao leiaute do mobiliário; sobreposição de usos em alguns ambientes; ausência do ambiente (não foi previsto no programa de necessidades da unidade); acessibilidade comprometida para pessoas com deficiência; e estado de conservação ruim de acabamentos e móveis, associado à falta de manutenção.

No que se refere ao subdimensionamento, verificou-se que muitos ambientes analisados não apresentavam a área mínima prevista na Resolução RDC 50/2002 (AGÊNCIA..., 2002), incluindo: salas de emergências, salas de exame indiferenciado (consultórios), salas de aplicação de medicamentos e salas de triagem. Nesse contexto destacam-se as salas de emergência, que frequentemente estavam abaixo da área mínima normatizada e que não conseguiam acomodar apropriadamente toda a demanda a ser atendida. No entanto, na maioria das situações, os ambientes analisados estavam apenas um pouco abaixo da área mínima normatizada. De qualquer modo, chama a atenção o fato de se tratarem de ambientes centrais para o atendimento de unidades de urgência e emergência.

Em outras situações verificou-se que os ambientes atendiam a Resolução RDC 50/2002 (AGÊNCIA..., 2002), mas ainda eram considerados subdimensionados pelos profissionais que atuavam no local, considerandose a demanda e as atividades realizadas. Nesses casos observou-se que a área mínima prevista na resolução nem sempre é suficiente para assegurar a qualidade ambiental desejável à sua vivência. Isso possivelmente se deve ao fato de que a resolução exige realmente uma área mínima e não necessariamente assegura a área desejável para contemplar todas as necessidades de uso. Por exemplo, a área mínima prevista na resolução para consultórios médicos permite acomodar as atividades de atendimento à saúde que lá ocorrem, mas torna difícil acomodar um local privativo para a troca de roupa do paciente para fins de exame, o que pode gerar constrangimento. 
Figura 4 - Parte do quadro síntese dos resultados obtidos com a aplicação do checklist 1

\begin{tabular}{|c|c|c|c|c|c|c|}
\hline & Hospital 01 & Hospital 02 & Hospital 03 & Hospital 04 & Hospital 05 & Hospital 06 \\
\hline $\begin{array}{c}\text { Area de } \\
\text { desemba rque } \\
\text { de ambulância }\end{array}$ & $\begin{array}{c}\text { Manutenção (porta de } \\
\text { acesso apresentava } \\
\text { problema) }\end{array}$ & $\begin{array}{c}\text { Programa de } \\
\text { necessidades (sem } \\
\text { area para desem barque } \\
\text { adequado para quem } \\
\text { chega por conta } \\
\text { própria) }\end{array}$ & \begin{tabular}{|c|} 
Manutenção \\
(revestimento e estado \\
de conservação \\
inadequado); não perm ite \\
manobra de ambulância; \\
e não apresenta \\
cobertura suficiente \\
\end{tabular} & $\begin{array}{l}\text { Não apresenta } \\
\text { problemas }\end{array}$ & $\begin{array}{l}\text { Sobreposição de usos; } \\
\text { acessibilidade (piso } \\
\text { irregular) }\end{array}$ & $\begin{array}{l}\text { Não apresenta } \\
\text { problemas }\end{array}$ \\
\hline Sala de espera & $\begin{array}{c}\text { Flexibilidade de projeto } \\
\text { (demanda maior que a } \\
\text { prevista em projeto) }\end{array}$ & $\begin{array}{l}\text { Mobiliário insuíciente } \\
\text { para a demanda }\end{array}$ & $\begin{array}{l}\text { Manutenção (bcos de } \\
\text { mofo), e mobiliário } \\
\text { insuiciente para a } \\
\text { demanda }\end{array}$ & $\begin{array}{l}\text { Não apresenta } \\
\text { problemas }\end{array}$ & $\begin{array}{l}\text { Layout; programa de } \\
\text { necessidades (não há } \\
\text { área para guarda de } \\
\text { maca e cadeira de } \\
\text { rodas); e manutenção }\end{array}$ & $\begin{array}{l}\text { Flexibilidade (demanda } \\
\text { maior que a prevista em } \\
\text { projeto) }\end{array}$ \\
\hline $\begin{array}{l}\text { Sala de espera } \\
\text { intermediária }\end{array}$ & $\begin{array}{c}\text { Sobreposição de uso } \\
\text { (sene também como } \\
\text { depósito) }\end{array}$ & Não existe na unidade & Não existe na unidade & $\begin{array}{c}\text { Programa de } \\
\text { necessidades } \\
\text { (caráter improvisado); } \\
\text { alta de mobiliário } \\
\text { adequado e } \\
\text { subdim ensionamento } \\
\text { (área útil insuiciente) } \\
\end{array}$ & $\begin{array}{c}\text { Programa de } \\
\text { necessidade (cárater } \\
\text { improvisado); ausência } \\
\text { de mobiliário adequado }\end{array}$ & $\begin{array}{l}\text { Não apresenta } \\
\text { problemas }\end{array}$ \\
\hline
\end{tabular}

Outro exemplo: a sala de emergências também foi com frequência considerada subdimensionada, mesmo quando contemplava a área mínima, relativa a dois leitos de atendimento. Isso ocorre porque em muitas dessas unidades é comum que sejam incorporados mais do que dois leitos de atendimento, visando atender a crescente demanda, comprometendo as áreas de circulação na sala de emergências e consequentemente a atuação da equipe de saúde. Cada unidade encontra-se em um contexto distinto, com uma demanda de atendimento específica que necessita ser previamente analisada e compreendida para dar suporte ao seu planejamento.

Por fim, verifica-se que o dimensionamento mínimo nem sempre será adequado para qualquer configuração espacial. Retomando o exemplo dos consultórios, observou-se nas unidades analisadas que quando esses ambientes apresentavam duas portas de acesso, visando facilitar o fluxo dos pacientes na unidade, tornava-se ainda mais difícil acomodar todo o mobiliário e as atividades necessárias na área mínima normatizada. A existência das duas portas aumenta a área de circulação interna do ambiente e reduz a área de paredes disponíveis para posicionar o mobiliário. Logo, evidencia-se que o dimensionamento correto pode ser comprometido pela configuração ambiental e pelo leiaute. Mesmo com dimensões reduzidas, se o leiaute fosse planejado de forma mais ajustada às demais características do ambiente, possivelmente seriam minimizados os prejuízos às atividades realizadas. Assim, destacase a importância de se considerar todas as características espaciais em conjunto, pois elas são interdependentes.

Entende-se que a resolução deve mesmo exigir áreas mínimas, pois de outro modo ela poderia se tornar muito restritiva, considerando-se que será aplicada em uma variedade muito grande de estabelecimentos de saúde e em todo o território nacional. Porém, os arquitetos que planejam as unidades de urgência e emergência precisam ter clareza das demandas, atividades e contexto de inserção de cada unidade para o seu correto dimensionamento. Deve-se, sempre que possível, dar preferência a áreas superiores ao mínimo exigido, especialmente nos ambientes supracitados que costumam ser centrais para o atendimento. Ao dimensionar ambientes com alguma folga na sua metragem quadrada em relação à Resolução RDC 50/2002 (AGÊNCIA..., 2002), torna-se mais fácil contemplar aspectos relativos à acessibilidade e à privacidade, pois se permite manter uma distância mínima e dispor de divisórias entre leitos e poltronas de pacientes. Torna-se então mais fácil a realização de uma maior diversidade de atividades de atendimento em um mesmo local, além das atividades para as quais $o$ ambiente foi inicialmente planejado.

Além disso, a maioria das unidades analisadas se encontra em hospitais públicos com mais de três décadas de existência. Durante as entrevistas, os funcionários comentaram que a crescente demanda 
de atendimento faz com que o dimensionamento previsto para alguns ambientes se torne insuficiente com o passar do tempo, ainda que fosse adequado no momento de sua inauguração. Para agravar a situação, muitos pacientes, que não são efetivamente casos de urgência e emergência, buscam essas unidades como porta de entrada para o sistema público de saúde, e pacientes atendidos que deveriam ser transferidos para unidades de internação hospitalar acabam permanecendo no local por falta de leitos disponíveis na rede pública. Como consequência, tem-se o agravamento do quadro de: subdimensionamento, sobreposição de usos em alguns ambientes - como quando os pacientes encontram-se hospitalizados nos corredores da unidade -, inadequação do leiaute pelo excesso de equipamentos e mobiliário, e comprometimento da acessibilidade. Tais problemas se mostraram recorrentes em: salas de espera, salas de observação, salas de emergências, salas de aplicação de medicamentos e corredores (Figura 5). Desse modo, as unidades situadas em hospitais públicos deveriam ser planejadas com critérios como flexibilidade interna, favorecendo a realização de reformas e ajustes, e expansibilidade, permitindo acomodar o aumento da demanda ao longo do tempo. A expansibilidade é um critério central em projetos de habitação popular, e também deveria ser um critério central no planejamento de edifícios hospitalares. Quando essa possibilidade não é prevista no projeto arquitetônico original, tende-se a improvisar soluções como aumentar o número de poltronas de atendimento em salas de aplicação de medicamentos, o que as tornam superlotadas e compromete a qualidade de sua vivência.
Nas unidades avaliadas, alguns ambientes obrigatórios segundo a Resolução RDC 50/2002 (AGÊNCIA..., 2002) para unidades de urgência e emergência de alta complexidade não foram previstos no programa de necessidades durante seu planejamento como: sala de inalação, sala de observação, sala de serviço social, sala de serviços e depósito de equipamentos. Além disso, também foi recorrente a ausência de alguns ambientes não obrigatórios como: sala de espera intermediária (uma segunda sala de espera na unidade), sala de exames diferenciados, sala de reidratação, e área de higienização.

Alguns desses ambientes realmente não são considerados indispensáveis para a unidade na percepção dos seus profissionais de saúde como: salas de exame diferenciado - consultórios de especialidades como ginecologia ou oftalmologia, cuja necessidade se fará sentir apenas em contextos específicos; sala de higienização, a qual costuma ser realizada dentro da própria sala de emergências, visando proporcionar rapidez no atendimento; e sala de serviço social. Embora a sala de serviço social seja obrigatória segundo a resolução, na maioria das unidades analisadas ela se encontrava em outro setor do hospital, e, sempre que necessário, os profissionais eram acionados e vinham até a unidade. Segundo os entrevistados, a posição dessa sala fora da unidade não causava prejuízo algum à qualidade do atendimento. Assim, considerando-se a percepção da equipe de saúde, a previsão desses ambientes no planejamento das unidades de urgência e emergência poderia, portanto, ser flexibilizada com a revisão da Resolução RDC 50/2002 (AGÊNCIA..., 2002).

\section{Figura 5 - Corredor de uma das unidades analisadas funcionando como internação improvisada}

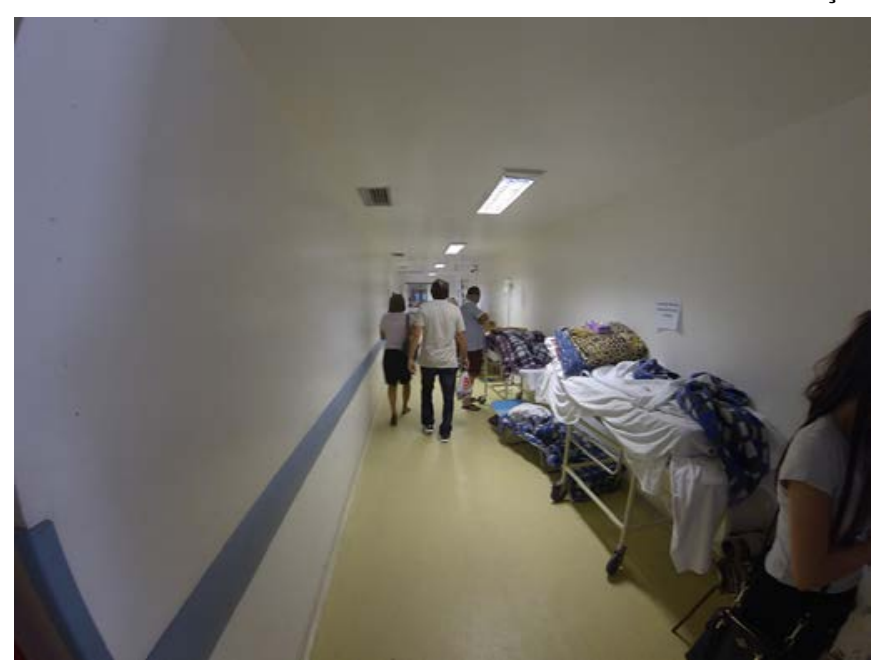


As atividades de inalação, reidratação e observação são centrais para a qualidade do atendimento. No entanto, embora as salas de inalação e de observação sejam ambientes obrigatórios segundo a resolução, em mais de uma unidade avaliada os profissionais de saúde apontaram que é comum que essas atividades ocorram em um único ambiente. Na percepção da equipe de saúde, concentrar as atividades em um único ambiente facilita sua atuação e a assistência ao paciente. Os profissionais de saúde participantes do estudo sugeriram dispor de mais de uma sala de aplicação de medicação quando a demanda de atendimento for alta, de forma a separar os pacientes de maior e menor gravidade, mas mantendo o perfil multiuso do ambiente. Em caso de ambientes multiusos, é importante que os arquitetos considerem a exigência normativa mais restritiva em termos de dimensionamento, de modo que a área disponível por leito seja realmente adequada a qualquer uma dessas atividades, o que nem sempre se verificou nos locais avaliados. Assim, na percepção dos entrevistados, no caso desses ambientes específicos o problema não seria a sobreposição de usos, mas o subdimensionamento do local frente à diversidade de atividades realizadas.

Nas unidades que dispõem de uma segunda sala de espera, verificou-se que ela permite acomodar separadamente os pacientes que já foram triados e que constituem os casos de maior urgência de atendimento (Figuras 6 e 7). A presença desse ambiente contribui para facilitar a atuação dos profissionais de saúde ao aproximar os casos mais graves dos locais onde se dará o atendimento, favorecendo o contato visual entre a equipe de saúde e os pacientes. Além disso, ao separar os casos mais graves dos demais, ameniza-se a tensão decorrente da demora de atendimento por parte de acompanhantes e pacientes em situação de menor gravidade ou risco. Essa separação também pode contribuir para evitar o contágio entre pacientes com patologias mais graves e os demais, no que se refere a doenças de fácil transmissão. A presença de uma segunda sala de espera realmente não deve ser obrigatória na resolução, mas mostrou-se como uma solução muito eficiente, e que poderia ser adotada no planejamento de unidades de médio a grande porte, visando contribuir para a atuação profissional e para uma experiência mais positiva do ambiente por todos.

Em boa parte das unidades analisadas faltam áreas de depósito bem dimensionadas e posicionadas como depósito de material de limpeza (DML), depósito de equipamentos e área para guarda de maca e cadeira de rodas. Quando esses ambientes não foram previstos no projeto, ou estão subdimensionados ou mal posicionados, observam-se equipamentos e caixas com produtos dispostos nas salas de espera (Figura 8), corredores ou outros locais da unidade. Isso ocasiona sobreposição de usos, e por vezes compromete a acessibilidade, o leiaute. Não dispor de depósitos em quantidade suficiente para a demanda foi um problema frequente nas unidades analisadas. É possível que tais ambientes não sejam priorizados durante o processo de planejamento por não serem locais de atendimento médico e de enfermagem. No entanto, destaca-se aqui sua importância, já que eles são estratégicos enquanto apoio logístico, o que é evidenciado pelo impacto negativo gerado por sua ausência em diversos outros ambientes da unidade.

Figura 6 - Sala de espera externa de Unidade que também apresenta uma sala de espera interna

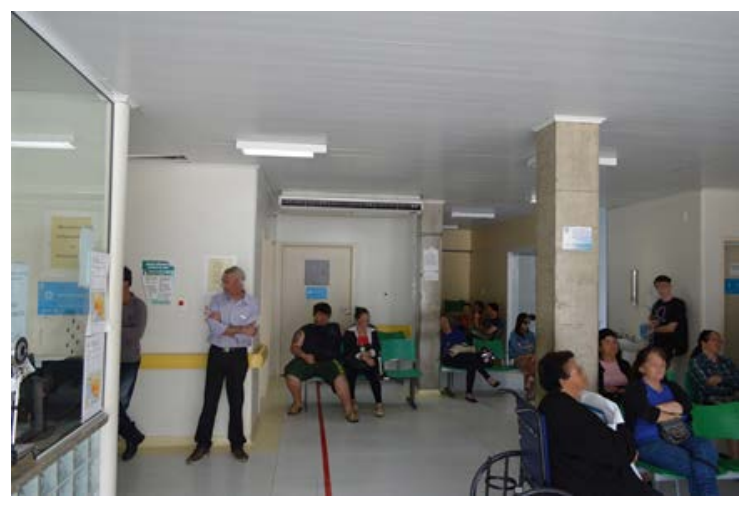

180 Cavalcanti, P. B.; Bins Ely, V. H. M.; J eremias, D. M.; Schneider, J. P.; Silva, M. V. da; Bortoluzzi, T. V. C. 
Figura 7 - Salas de espera interna a qual favorece a separação dos pacientes por gravidade

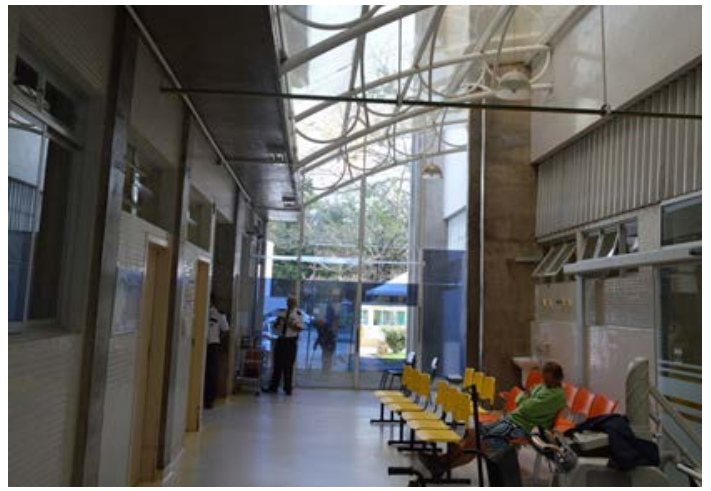

Figura 8 - Equipamentos armazenados junto à sala de espera

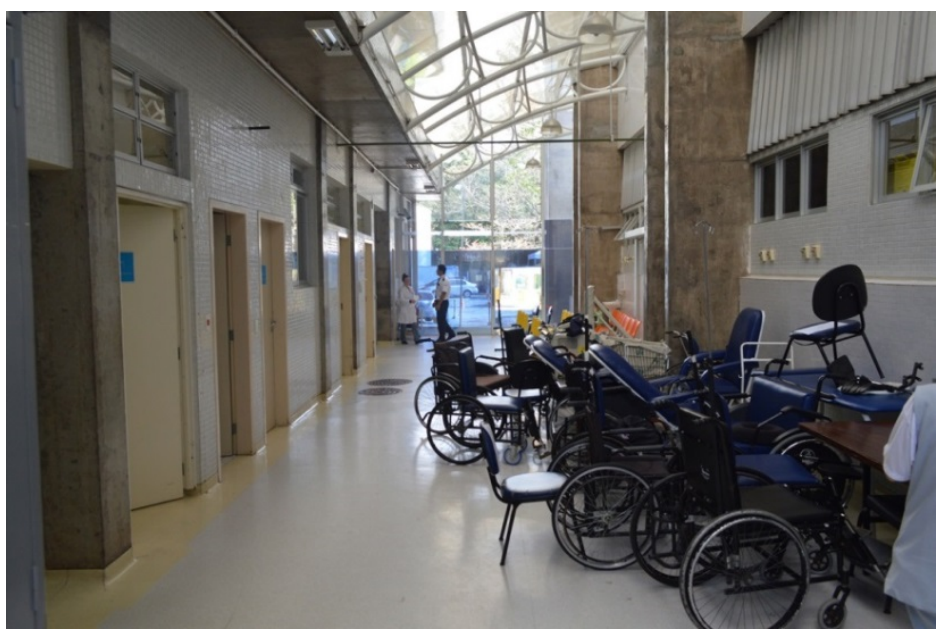

A previsão de salas de isolamento no programa de necessidades não é obrigatória na resolução, nem mesmo para urgências e emergências de alta complexidade. Porém, elas estavam presentes em todas as unidades analisadas, possivelmente porque sua necessidade se fez sentir. No entanto, por não ser obrigatória segundo a resolução, a maioria desses ambientes não apresentava todas as condições necessárias para assegurar o isolamento de pacientes, e parte deles se encontrava funcionando em algum local adaptado para atender essa demanda. Em dois dos hospitais analisados, as unidades foram projetadas com quartos de isolamento, que foram posteriormente utilizados para outro fim, e acabou-se adaptando um terceiro ambiente para funcionar novamente como quarto de isolamento. Apenas em um dos hospitais analisados a sala de isolamento contemplava a maioria dos aspectos desejáveis ao ambiente (antecâmara ou área de paramentação, sistema adequado de exaustão do ar com pressão positiva e negativa, visores, lavatório e banheiro próprio). As salas de isolamento são fundamentais tanto para acomodar pacientes com doenças infecciosas de fácil transmissão quanto para acomodar pacientes imunodeprimidos. Desse modo, levanta-se aqui o questionamento, a ser aprofundado em estudos futuros, sobre a possibilidade de tornar obrigatória segundo a resolução a previsão de ao menos um quarto de isolamento por unidade de urgência $e$ emergência geral de alta complexidade situada em hospital de médio a grande porte.

Além dos problemas já descritos, observaram-se ainda:

(a) ausência de equipamentos, móveis ou objetos que seriam desejáveis para dar suporte às atividades realizadas como armário para guarda dos pertences pessoais dos pacientes;

(b) revestimentos de pisos, paredes, forros e mobiliário em estado precário de conservação e de manutenção;

(c) problemas de acessibilidade em sanitários e banheiros. Como muitas das unidades foram executadas antes da publicação das primeiras normas de acessibilidade, com frequência estes ambientes não estão adequadamente dimensionados para o uso de cadeiras de rodas e outros equipamentos; e 
(d) dependência de sistema de climatização artificial para proporcionar conforto térmico, seja pela falta de aberturas ou, quando estas existiam, são mantidas fechadas por motivos de proximidade excessiva a outros prédios, proximidade a locais onde pessoas fumavam ou conversavam alto, ou ainda por não serem suficientes para proporcionar a renovação adequada do ar e o conforto térmico. Desse modo, os usuários tendiam a usar a climatização artificial por meio do arcondicionado, frequentemente aparelhos do tipo split. Porém, se houvesse no hospital acesso fácil a jardins que os usuários pudessem utilizar para conversar e descansar, e locais próprios para fumantes situados longe de janelas de ambientes de atendimento, isso favoreceria a utilização da ventilação natural nessas unidades. Nas circunstâncias atuais, se houver algum problema com o funcionamento dos aparelhos de arcondicionado tende-se a vivenciar desconforto térmico e renovação inadequada do ar. Além disso, deixa-se de dispor de um melhor aproveitamento dos recursos naturais e financeiros pela incorporação de estratégias de sustentabilidade ao projeto da edificação.

Por fim, cabe destacar que o mobiliário antigo associado a problemas frequentes de manutenção do ambiente, como mofo, cupim e acabamentos deteriorados, compromete a ambiência das unidades analisadas. Apenas uma das unidades, recentemente reformada, não se enquadra na descrição acima. Porém, mesmo nessa unidade, embora os móveis sejam novos e estejam em excelente estado de conservação, eles foram avaliados pela equipe de saúde como desconfortáveis. Assim, embora muitos desses hospitais sejam referências em distintas áreas de atendimento à saúde, pelo elevado padrão de atendimento e serviços prestados, sua imagem não contribui para que eles se mostrem acolhedores e amigáveis para seus usuários.

No quesito humanização, observa-se ainda: a ausência de privacidade em salas de observação, as quais nem sempre apresentavam cortinas hospitalares ou divisórias entre os leitos; a ausência de local para troca de roupa em consultórios, já mencionada; além da falta de local para guarda de pertences dos pacientes. Pela falta de divisórias e de compartimentação entre leitos em ambientes coletivos, raramente os pacientes terão algum controle sobre as condições ambientais como poder regular o volume do som e o nível de iluminação incidente. Na maioria das unidades também não havia acomodações adequadas para os acompanhantes em diversos ambientes, destacando-se sua importância em salas de observação e de aplicação de medicação, que são locais de permanência mais prolongada. Por fim, faltam ainda distrações positivas - como integração interior-exterior, vegetação, obras de arte e revisteiros - as quais poderiam contribuir para amenizar a percepção de dor pelo paciente e a sensação de demora no atendimento.

\section{Considerações finais}

Ao longo da pesquisa foi possível realizar uma avaliação preliminar do espaço físico das unidades de urgência e emergência de hospitais públicos da Grande Florianópolis. A revisão de literatura, as duas visitas exploratórias e os seis estudos de caso realizados permitiram dispor de uma visão abrangente das unidades do município, identificando problemas recorrentes em sua configuração ambiental e alguns aspectos desejáveis a seu planejamento.

Dentre os principais problemas recorrentes nas unidades de urgência e emergência avaliadas estão: subdimensionamento de ambientes, inadequação do leiaute, sobreposição de usos, ausência de ambientes, falta de manutenção e condições inadequadas de acessibilidade. Para evitar que tais problemas venham a ocorrer em projetos futuros algumas medidas poderiam ser tomadas em três âmbitos distintos: planejamento arquitetônico; alteração da RDC 50/2002 (AGÊNCIA..., 2002), a qual já está em revisão; e políticas públicas relativas à gestão e planejamento dos estabelecimentos assistenciais de saúde.

Observou-se que a grande maioria das exigências da RDC 50/2002 (AGÊNCIA..., 2002) para unidades de urgência e emergência realmente refere-se a aspectos mínimos a serem assegurados pela configuração ambiental. Assim, acredita-se que boa parte dos problemas constatados não demanda a revisão da resolução, mas que poderiam ser evitados por meio de algumas estratégias de projeto incorporadas ao planejamento dessas unidades pelos arquitetos responsáveis. Uma dessas estratégias projetuais é o dimensionamento adequado de ambientes centrais ao atendimento, considerando as necessidades efetivas de seus usuários. Em muitas situações analisadas, o atendimento à área mínima normatizada não foi suficiente para proporcionar a satisfação dos usuários. Um dimensionamento apropriado deve considerar: a acomodação da diversidade de atividades e demanda real de usuários, o leiaute de móveis e equipamentos, a acessibilidade para cadeiras de rodas e macas, a privacidade - pelo espaçamento maior entre poltronas e leitos de atendimento e o uso de divisórias -, entre outros.

Outro aspecto que merece atenção dos arquitetos no planejamento dessas unidades é a flexibilidade 
interna e expansibilidade para realização de reformas e ampliações. As reformas são necessárias nos diversos setores de um estabelecimento de saúde no intuito de permitir sua adequação a novos modos de atendimento e a incorporação de novas tecnologias, evitando sua obsolescência precoce. As ampliações também se mostraram uma necessidade recorrente nas unidades analisadas, visto que a construção de novos hospitais aparentemente não tem sido realizada em quantidade suficiente para acompanhar a crescente demanda de atendimento da rede pública.

Por fim, sempre que possível, sugere-se incluir no planejamento da unidade: salas de espera distintas para os diferentes perfis usuários; depósitos em quantidade e dimensões suficientes; e salas exclusivas para inalação, reidratação, aplicação de medicamentos e observação ou então dimensionar apropriadamente as salas de aplicação de medicamentos caso elas sejam multiuso. Observou-se que os depósitos tendem a ser negligenciados no planejamento, e que, em sua ausência, insumos, materiais de limpeza e equipamentos serão armazenados inapropriadamente em outros ambientes da unidade, dificultando a circulação e a realização das atividades de atendimento.

No âmbito da legislação, há algumas alterações que poderiam ser discutidas no processo de revisão da RDC 50/2002 (AGÊNCIA..., 2002). Neste estudo, e segundo a percepção dos profissionais de saúde entrevistados, as salas de serviço social não precisariam obrigatoriamente ser previstas na unidade, pois em muitos hospitais elas localizamse em outras alas e isso não compromete a qualidade do atendimento. Por outro lado, as salas de isolamento merecem estudos futuros que confirmem a necessidade de sua obrigatoriedade. Embora atualmente as salas de isolamento não sejam obrigatórias, elas estavam presentes em todas as unidades avaliadas, e, em muitos casos foram fruto de adaptações e reformas que as tornavam apenas parcialmente adequadas a essa atividade. Ao menos em unidades de urgência e emergência de média a alta complexidade de hospitais de médio a grande porte mostra-se desejável prever as salas de isolamento para que se assegurem: menor risco de disseminação de doenças e melhores condições de atendimento e enfrentamento de epidemias.

Por fim, observa-se ainda que parte dos problemas constatados encontra-se no âmbito da administração pública, pois não são relativos às normas vigentes ou à atuação dos arquitetos. Tais problemas referem-se às políticas públicas como um todo, que precisam assegurar o bom funcionamento do sistema público de saúde. Assim, ainda que uma unidade de urgência e emergência seja planejada de forma apropriada, é preciso assegurar sua manutenção e conservação em longo prazo. Também é necessário: investir em políticas de saúde de prevenção de doenças e melhorar a capacidade de atendimento na rede primária e secundária, de forma a reduzir a procura pelas unidades de urgência e emergência hospitalares; e promover a conscientização da população sobre a função dessas unidades, evitando que elas sejam utilizadas indiscriminadamente como uma das principais formas de acesso à rede pública de saúde. Apenas por meio de políticas públicas poder-se-ia alterar esse cenário, proporcionando melhores condições de atendimento ambulatorial e preventivo à saúde, educando a população quanto à função desempenhada por essa unidade, e evitando a sua superlotação. Também é preciso assegurar que haja leitos de internação suficientes nos hospitais públicos para receber as pessoas que já foram atendidas nas unidades de urgência e emergência, mas que necessitam seguir hospitalizadas. Faz-se ainda necessário considerar a adequada expansão da rede pública de saúde de forma a garantir a construção de novas unidades de urgência e emergência em quantidade coerente com o crescimento da demanda por atendimento. Assim, não apenas deve-se planejar as unidades de urgência e emergência considerando a possibilidade de expansão, como é preciso planejar a rede pública de saúde visando sua ampliação no médio e longo prazo. A construção de novos estabelecimentos de saúde, quando necessários, contribuiria para a descentralização do atendimento, aproximando-o da comunidade a ser atendida. Desse modo, os hospitais existentes não seriam pressionados a ampliar-se indefinidamente, o que repercute na descaracterização e no comprometimento da qualidade de sua arquitetura. A ampliação contínua faz com que os hospitais de grande porte se configurem como estabelecimentos muito grandes e difíceis de gerenciar, impactantes na paisagem do entorno e distantes da escala humana.

O presente estudo evidenciou ainda que a humanização da arquitetura hospitalar, que vem sendo amplamente discutida em pesquisas científicas nacionais e internacionais desde os anos 1980, ainda é incipiente nas unidades analisadas. Tais estabelecimentos enfrentam problemas consideráveis como a falta de manutenção e conservação, que comprometem sua ambiência. Além disso, mesmo estratégias projetuais de baixo custo são ainda pouco exploradas, como: a 
integração interior-exterior, ventilação e iluminação natural, e algumas distrações positivas.

Acredita-se que a abordagem metodológica permitiu ter uma visão abrangente das unidades, pois embora se tratasse de um único método análise Walkthrough - ele se baseou em um roteiro extenso e que abarcava a percepção da equipe de saúde, além da avaliação técnica dos próprios pesquisadores. De qualquer modo, estudos futuros com abordagens metodológicas diferentes poderão trazer novos olhares sobre o planejamento dessas unidades.

Além disso, os estudos de caso foram realizados exclusivamente em unidades de urgência e emergência gerais, para atendimento de adultos ou crianças, de hospitais públicos de Florianópolis. Assim, mostram-se necessários novos estudos que possam avaliar a realidade de unidades de urgência e emergência de outras localidades do país, e ainda de unidades especializadas como aquelas destinadas à psiquiatria ou à cardiologia, evidenciando suas especificidades.

A escassez de pesquisas realizadas na área hospitalar e no contexto brasileiro, somada à complexidade e a constante evolução dessas edificações, torna necessário realizar estudos contínuos que tragam suporte a seu planejamento. De qualquer modo, espera-se que os resultados deste trabalho possam contribuir para: a reflexão sobre o tema; auxiliar os órgãos responsáveis pela legislação, planejamento e manutenção dessas unidades; e auxiliar os arquitetos que atuam na área, dando-lhes suporte na elaboração de projetos futuros. Espera-se ainda que os checklists desenvolvidos possam ser utilizados para avaliação de unidades em todo o país, contribuindo para o planejamento de obras de reforma ou ampliação e permitindo criar um panorama geral dessas unidades por região geográfica - municípios e estados.

\section{Referências}

ACOSTA, A. M.; LIMA, M. A. D. S. Frequent Users of Emergency Services: associated factors and reasons for seeking care. Revista LatinoAmericana de Enfermagem, v. 23, n. 2, p. 337344, abr. 2015.

\section{AGÊNCIA NACIONAL DE VIGILÂNCIA}

SANITÁRIA. Resolução RDC 50/2002, que dispõe sobre o regulamento técnico para planejamento, programação, elaboração e avaliação de projetos físicos de Estabelecimentos Assistenciais de saúde. Diário Oficial da União, Brasília, 2002.
ALMEIDA, C. et al. Health Sector Reform in Brazil: a case study of inequity. International Journal of Health Services: Planning, Administration, Evaluation, v. 30, n. 1, p. 129162, 2000.

\section{ASSOCIAÇÃO BRASILEIRA DE NORMAS}

TÉCNICAS. NBR 9050: acessibilidade a edificações, mobiliário, espaços e equipamentos Urbanos. Rio de Janeiro, 2004.

\section{ASSOCIAÇÃO BRASILEIRA DE NORMAS}

TÉCNICAS. NBR 9050: acessibilidade a edificações, mobiliário, espaços e equipamentos Urbanos. Rio de Janeiro, 2015.

AZEVEDO, A. L. C. S. et al. Organização de serviços de Emergência Hospitalar: uma revisão integrativa de pesquisas. Revista Eletrônica de Enfermagem, v. 12, n. 4, p. 736-45, 2010.

BRASIL. Ministério da Saúde. Política Nacional de Atenção às Urgências. Brasília, 2006.

Disponível em:

<http://bvsms.saude.gov.br/bvs/publicacoes/politic a_nacional_atencao_urgencias_3ed.pdf $>$. Acesso em: jan. 2017.

BRASIL. Ministério da Saúde. Portaria no 2.048, de 5 de novembro de 2002. Disponível em:

<http://bvsms.saude.gov.br/bvs/saudelegis/gm/200 2/prt2048_05_11_2002.html>. Acesso em: $3 \mathrm{dez}$. 2016.

BRASIL. Ministério da Saúde. Secretaria de Atenção à Saúde. Departamento de Atenção Especializada. Manual Instrutivo da Rede de Atenção às Urgências e Emergências no Sistema Único de Saúde (SUS). Brasília, 2013. Disponível em:

<http://bvsms.saude.gov.br/bvs/publicacoes/manua 1_instrutivo_rede_atencao_urgencias.pdf $>$. Acesso em: jan. 2017.

DERLET, R. W.; RICHARDS, J. R.

Overcrowding in the Nation's Emergency

Departments: complex causes and disturbing effects. Annals of Emergency Medicine, Sacramento, v. 35, n. 1, p. 63-68, 2000.

DESLANDES, S. F. et al. Diagnostic Characterization of Services Providing Care to Victims of Accidents and Violence in Five Brazilian State Capitals. Ciência \& Saúde Coletiva, Rio de Janeiro, v. 11, n. 2, p. 385-396, jun. 2006.

FATOVICH, D. M. Emergency Medicine. British Medical Journal, v. 324, n. 7343, p. 958-962, 2002. 
GAWRYSZEWSKI, V. P.; KOIZUMI, M. S.; MELLO-JORGE, M. H. P. As Causas Externas no Brasil no Ano 2000: comparando a mortalidade e a morbidade. Cadernos de Saúde Pública, Rio de Janeiro, v. 20, n. 4, p. 995-1003, aug. 2004.

GIACOMO, N. S. Diretrizes Projetuais Para Unidades de Urgência e Emergência Hospitalares Eficientes. São Paulo, 2011. Tese (Doutorado em Projeto de Arquitetura) - Faculdade de Arquitetura e Urbanismo, Universidade de São Paulo, São Paulo, 2011.

GÓES, R. Manual Prático de Arquitetura Para Clínicas e Laboratórios. São Paulo: Edgar Blücher, 2006.

JEREMIAS, D. M. et al. Avaliação Arquitetônica de Uma Unidade de Urgência e Emergência.

Ambiente Hospitalar - Revista Interdisciplinar de Infraestrutura em Saúde, São Paulo, v. 11, p. 17-25, 2017.

NATIONAL HEALTH SYSTEM. AEDET

Evolution: Achieving Excellence Design

Evaluation Toolkit - Instructions, Scoring and Guidance. 2005a. Disponível em:

$<$ http://www.dh.gov.uk/en/Procurementandpropos als/Publicprivatepartnership/Privatefinanceinitiativ e/ InvestmentGuidanceRouteMap/DH_4132945>. Acesso em: 31 mar. 2009.

NATIONAL HEALTH SYSTEM. ASPECT: Staff and Patient Environment Calibration Toolkit 21. 2005b. Disponível em:

$<$ http://www.dh.gov.uk/en/Publicationsandstatistic s/Publications/ PublicationsPolicyAndGuidance/ DH_082087>. Acesso em: 31 mar. 2009.

OLIVEIRA, T. A.; PINTO, K. A. Acolhimento Com Classificação de Risco e Acesso em Serviço de Emergência: avaliação de usuários. Ciência, Cuidado e Saúde, Londrina, v. 14, n. 2, p. 1.1221.129, 2015.

ORGANIZAÇÃO MUNDIAL DA SAÚDE. Declaração de Alma-Ata. Alma-Ata: OMS, 1978.

PASCALE, F. et al. Evaluation of Factors and Approaches Affecting Emergency Department Space Planning. Facilities, v. 32, n. 13/14, p. 761785, 2014.
PAUL, S. A.; REDDY, M. C.; DEFLITCH, C. J. A. Systematic Review of Simulation Studies Investigating Emergency Department Overcrowding. Simulation, v. 86, n. 8-9, p. 559571, 2010.

POLL, M. A.; LUNARDI, V. L.; LUNARDI FILHO, W. D. Healthcare in Emergency Units: organization and ethical implications. Acta Paulista de Enfermagem, v. 21, n. 3, p. 509-514, 2008.

PUCCINI, P. T.; CORNETTA, V. K. Ocorrências em Pronto-Socorro: eventos sentinela para o monitoramento da atenção básica de saúde. Cadernos de Saúde Pública, Rio de Janeiro, v. 24, n. 9, p. 2.032-2.042, 2008.

RHEINGANTZ, P. A. et al. Observando a Qualidade do Lugar: procedimentos para a avaliação pós-ocupação. Rio de Janeiro: Universidade Federal do Rio de Janeiro, 2009.

RISMANCHIAN, F.; HOON LEE, Y. H. Process Mining-Based Method of Designing and Optimizing the Layouts of Emergency Departments in Hospitals. HEARD: Health Environments Research \& Design Journal, v. 10, n. 4, p. 105-120, 2017.

TOLEDO, L. C.; FERRER, M. V. Urgência e Emergência: primeiros cuidados projetuais. In: CARVALHO, A. P. A. (Org.). Arquitetura de Unidades Hospitalares. Salvador: FAUFBA, ARQSAUDE/GEA-hosp, ISC, 2004.

ZILM, F. A New Era of Emergency Care: planning and design consideration. Journal of Ambulatory Care Management, v. 30, n. 3, p. 259-63, 2007.

\section{Agradecimentos}

Agradecemos ao Ministério da Educação (MEC) e à Secretaria de Educação Superior (Sesu) que deu suporte para a realização da presente pesquisa por meio da participação de dois bolsistas do Programa de Educação Tutorial (PET). 


\section{Patrícia Biasi Cavalcanti}

Departamento de Expressão Gráfica | Universidade Federal de Santa Catarina | Rua Engenheiro Agronômico Andrei Cristian Ferreira, s/n, Campus Reitor J oão David Ferreira Lima, Trindade | Florianópolis - SC - Brasil | CEP 88040-900 | Tel.: (48)3721-3796 | E-mail: patibiasi@yahoo.com

\section{Vera Helena Moro Bins Ely}

Departamento de Arquitetura e Urbanismo | Universidade Federal de Santa Catarina | E-mail: vera.binsely@gmail.com

Diogo Mello J eremias

Departamento de Arquitetura e Urbanismo | Universidade Federal de Santa Catarina | E-mail: diogomellojeremias@gmail.com

J oão Pedro Schneider

Departamento de Arquitetura e Urbanismo | Universidade Federal de Santa Catarina | E-mail: joaopedrosndr@gmail.com

\section{Marcus Vinícius da Silva}

Departamento de Arquitetura e Urbanismo | Universidade Federal de Santa Catarina | E-mail: marcusvsilva@icloud.com

\section{Thaize Vanessa Costa Bortoluzzi}

Departamento de Arquitetura e Urbanismo | Universidade Federal de Santa Catarina | E-mail: thaizebortoluzzi@gmail.com

\section{Revista Ambiente Construído}

Associação Nacional de Tecnologia do Ambiente Construído
Av. Osvaldo Aranha, 99 - 3o andar, Centro
Porto Alegre - RS - Brasil
CEP $90035-190$
Telefone: +55 (51) 3308-4084
Fax: +55 (51) 3308-4054
www. seer. ufrgs. br/ ambienteconstruido
E-mail: ambienteconstruido@ufrgs.br

This is an open-access article distributed under the terms of the Creative Commons Attribution License.

186 Cavalcanti, P. B.; Bins Ely, V. H. M.; J eremias, D. M.; Schneider, J. P.; Silva, M. V. da; Bortoluzzi, T. V. C. 